\title{
Does Shear Thickening Occur in Semisolid Metals?
}

\author{
HELEN V. ATKINSON and VERONIQUE FAVIER
}

In the various forms of semisolid processing such as thixoforming and thixoforging, the entry into the die occurs in a fraction of a second so it is the transient rheological behavior which governs the initial stages of flow. In experiments in the literature, this rheological behavior is probed through applying rapid transitions in shear rate under isothermal conditions. There is contradictory evidence as to whether the behavior during these transitions is shear thinning or shear thickening, although it is clear that once in the die the material is thinning. Here the data in the literature are reanalyzed to obtain a rationalization of the contradictions which has not previously been available. It is argued that if a suspension is initially in a disagglomerated state (i.e., one which is initially sheared), the instantaneous behavior with a jump-up in shear rate is shear thickening (even if the long-term steady-state behavior is shear thinning) provided the fraction solid is greater than about 0.36 and the final shear rate at the end of the jump is greater than about $100 \mathrm{~s}^{-1}$. If the jump-up in shear rate is made from rest then yield masks the shear thickening.

DOI: $10.1007 / \mathrm{s} 11661-015-3307-4$

(C) The Author(s) 2016. This article is published with open access at Springerlink.com

\section{INTRODUCTION AND BACKGROUND}

PROCESSING of metals in the semisolid state is a widely established technology (e.g. ${ }^{[1]}$ Thixoforming, thixocasting, thixoforging, thixomolding, rheocasting, and rheoforming are all semisolid processing methods. They rely on the thixotropic property of metallic alloys in the semisolid state which have a spheroidal rather than dendritic microstructure; when sheared the material thins but when allowed to stand, it thickens again. ${ }^{[2,3]}$ This behavior is exploited to drive the material into a die and obtain a near net shape component in one shot. Although the technology is established, the modeling of such complex systems still needs further development. In particular, the entry into the die occurs in a fraction of a second so it is the transient rheological behavior which governs the initial flow. In the literature, this rheological behavior is probed experimentally through applying rapid transitions in shear rate under isothermal conditions.

In this section, the scene will be set for the reanalysis by outlining the basic nature of thixotropy in semisolid metallic alloy systems; the distinction between steadystate behavior and that associated with a transient; the connection between discontinuous shear thickening as encountered for dense suspensions of cornstarch in water and the potential for shear thickening to be

HELEN V. ATKINSON, Head of Department, is with the Department of Engineering, University of Leicester, University Rd., Leicester LE1 7RH, U.K. Contact e-mail: hva2@1e.ac.uk VERONIQUE FAVIER, Professor, is with the Arts et Métiers Paris Tech, PIMM, UMR, CNRS 8006, 151 Bd. De l'Hopital, 75013 Paris, France.

Manuscript submitted January 27, 2015.

Article published online January 20, 2016 masked by shear stress. In addition, the interplays between yield stress and thixotropy and the distinction between the existence of a yielded zone and shear banding/shear localisation are described. All these issues are relevant to the reanalysis here of the experimental data in the literature to arrive at a rationalization of the contradictory evidence as to whether shear thickening does occur in semisolid metals. Finally, in this section, the concept of 'isostructure' with rapid transitions in shear rate is highlighted along with the issues around the timescale for rapid shear rate jump experiments, before the aim of the paper is summarized.

\section{A. Basic Nature of Thixotropy in Semisolid Metallic Alloy Systems}

The particles here are spheroids of solid metal in a Newtonian liquid metal matrix and there is the potential for particle aggregation by the formation of minute 'welds' at the points where the spheroids contact by a mechanism very akin to sintering. The particles therefore show some cohesion in the terminology of soil mechanics. However, many of the mechanisms of interaction which apply in non-metallic suspension systems (e.g., electrostatic, steric, induced electric or magnetic dipoles) do not apply here. When the material is allowed to stand, or is sheared at a relatively low shear rate, the particles gradually agglomerate, with the average size of the agglomerates related to the shear rate. ${ }^{[3]}$ If the solid fraction is fairly high (in the region of 0.4 or above), when the material is still, the spheroids develop a solid 'skeleton' that provides some rigidity. The authors have recently shown that the skeleton introduces an elastic-type response into the behavior during rapid compression from rest. ${ }^{[4]}$ When the shear rate increases, the agglomerates are broken up and, for a constant shear rate, a new characteristic agglomerate 
size is established. Therefore, in the steady state at a given shear rate, there is an equilibrium between agglomeration and disagglomeration.

\section{B. Distinction Between Steady-State Behavior and Transients}

It is important to distinguish here between steadystate behavior and that associated with a transient. So for alloys in the partially solid state, if the material is sheared for a substantial period at a particular shear rate, a steady state will be established (although this may be a quasi-steady state because some particle coarsening will be occurring by Ostwald ripening). If a series of such steady states are established and the viscosity for each calculated, a plot of viscosity vs shear rate can be derived. When the viscosity decreases with increasing shear rate, the material is termed shear thinning and, if it increases, shear thickening. ${ }^{[5]}$ For alloys with spheroidal microstructure in the semisolid state, the behavior in the steady state is always shear thinning.

\section{Connection with Discontinuous Shear Thickening in Dense Suspensions of Cornstarch in Water: The Masking of Shear Thickening by a Yield Stress}

A particularly dramatic form of shear thickening is that termed discontinuous shear thickening where the viscosity increases by many orders of magnitude for a small increase in shear rate. This is the phenomenon observed with dense suspensions of cornstarch in water, which allows someone to run across the surface of the suspension in a swimming bath for example (but when they stand still they sink in). Brown et al. ${ }^{[6]}$ have argued that all suspensions should show shear thickening under certain conditions because the underlying mechanisms (hydrodynamics, ${ }^{[7,8]}$ dilatation ${ }^{[9-11]}$ ) are general but they demonstrate that shear thickening can be masked by a yield stress. They attribute discontinuous shear thickening to frictional particle contacts that form when dense particle arrangements begin to dilate and push against boundaries. ${ }^{[12]}$ The shear thickening could be enhanced to give a discontinuous effect if the hydroclusters (giving rise to continuous shear thickening) eventually become so large that they span the system and jam. Brown and Jaeger ${ }^{[13]}$ have recently identified for cornstarch in water that shear thickening can occur because of the confining stress at boundaries frustrating dilatation.

Micromechanical modeling applied on a Representative Volume Element containing solid globules, solid bonds, entrapped and free liquid has been developed and used to predict the strain rate sensitivity of the overall viscous material (Favier et al. ${ }^{[14]}$ ). The solid phases were considered non-Newtonian with a strain rate sensitivity index lower than one, as for hotdeformed alloys, while the liquid phases were considered as Newtonian. The modeling, considering viscous interactions, was not able to produce a shear-thickening behavior. This result is consistent with the fact that the shear thickening response is not an intrinsic bulk material response but is related to interaction with the boundaries which confine the suspension (Brown and Jaeger ${ }^{[15]}$ ).

\section{Existence of a Yielded Zone; Distinction Between a Yielded Zone and Shear Banding/Shear Localisation}

The interplay between yield stress and thixotropy is discussed in Møller et al. ${ }^{[16]}$ They argue that below a critical shear rate, all the flow is localized in a region near the shearing wall, and if the globally imposed shear rate is increased it is not the shear rate in the sheared region that increases but rather the extent of the sheared region which grows - to fill the entire gap of the shear cell exactly at the critical shear rate. (Note that this localization is distinct from wall slip). Møller et al. ', $S^{[16]}$ paper is concerned with the steady state rather than transients, and is supported by experimental data for semisolid metal alloys. ${ }^{[17]}$ Alexandrou et al. ${ }^{[18,19]}$ focus on the early stages of breakdown by applying finite element modeling to the fluid in the rheometer gap, presenting graphs which show, for shear rates below a critical shear rate, the yielded zone initially expands but then the boundary between yielded and unyielded material reaches a stationary position (and if the gap is relatively narrow this will be equivalent to the whole gap having yielded). In addition, the boundary can in fact retract.

There is a distinction between the existence of a yielded zone and issues of shear banding and shear localisation. Essentially, once shear localisation starts to occur, it becomes a self-exacerbating phenomenon with shear being increasingly concentrated in that band. Gourlay and Dahle have discussed this in a paper in Nature $^{[20]}$ with illustrations of banding from a vane rheometer (Figure 1(a)). However, in the quenched samples from concentric cylinder rheometers (e.g., Liu's thesis ${ }^{[21]}$, there is no evidence of such banding (Figure 1(b)). This may well be because the number of particles spanning the gap is much smaller than in the Gourlay and Dahle experiments. ${ }^{[20]}$ Also the Gourlay and Dahle hypothesis is essentially based around dilatation. In contrast with Gourlay and Dahle, in the concentric cylinder rheometer experiments reviewed here, there are relatively few particles spanning a gap which is long and narrow, giving severe restriction on dilatation. This is consistent with the argument in Brown and Jaeger ${ }^{[12]}$ about the confining stress of boundaries frustrating dilatation.

\section{E. The Concept of 'Isostructure' and the Timescale for Rapid Shear Rate Jump Experiments}

The behavior during such transient conditions of shear rate for semisolid alloys has been represented by Quaak $^{[22]}$ as shown in Figure 2. When a rapid jump-up in shear rate occurs (the upper part of Figure 2), immediately after the jump (in that instant), the material is 'isostructure' with the starting material. A fast process of deagglomeration (breaking of bonds between spheroids) then occurs. Subsequently, a much slower diffusion controlled process of coarsening and spheroidisation takes place (note the change in shape of particles). For a 
jump-down in shear rate (the lower part of Figure 2), the processes after 'isostructure' are 'fast' agglomeration followed by a slow growth of necks between spheroids and coarsening (although it should be noted that the agglomeration in the jump-down in the lower half of Figure 2 is significantly slower than the deagglomeration in the upper half with the jump-up).

A rapid jump-up in shear rate then has to be fast enough to ensure isostructure, i.e., it has to be faster than the characteristic disagglomeration time. Kumar

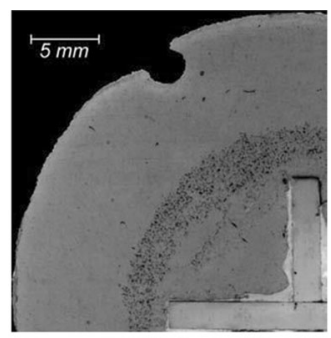

(a)

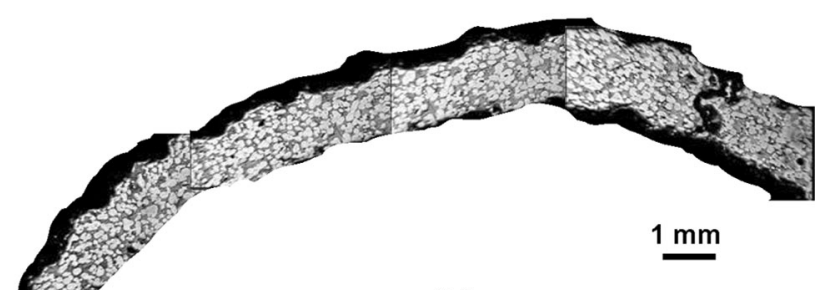

(b)

Fig. 1-(a) Vane rheometry of partially solidified $\mathrm{Mg}$ alloy showing shear banding evidenced by a localized band of porosity approximately 11 grains wide, taken with permission from Ref. [20]. (b) Sn 15 pct $\mathrm{Pb}$ alloy quenched in a cylindrical rheometer gap showing that no shear banding or discontinuity across the microstructure in the gap is evident, taken with permission from Ref. [21]. et $a .^{[23]}$ evaluate this to be of the order of seconds but Liu et al. ${ }^{[24]}$ find it to be in the region of $\sim 0.16 \mathrm{~s}$ (their Table I). The time for the transient in shear rate is of the order of $10 \mathrm{~ms}$ in Kumar et al. ${ }^{[23]}$ and $100 \mathrm{~ms}$ in Liu et $a .^{[24]}$ thus satisfying this requirement. The timescale for the transient in Koke and Modigell ${ }^{[25]}$ is not stated but is short. Peng and Wang ${ }^{[26]}$ have a transient time of $0.1 \mathrm{~s}$. In practice, a purely isostructural jump will be impossible to achieve because some microstructural processes will always start to occur during the jump.

\section{F. Aim of the Paper}

There is contradictory evidence in the literature ${ }^{[22-29]}$ as to whether the behavior during rapid increases in shear rate is shear thinning or shear thickening, although it is clear that once in the die the material is thinning. ${ }^{[30]}$ Kumar et al. ${ }^{[23,27]}$ and Koke and Modigell $^{[25,28]}$ (with further analysis of Reference 28 by Burgos et al. ${ }^{[29]}$ ) find their material to be shear thickening during the transient, Quaak ${ }^{[22]}$ and Liu et al. ${ }^{[24]}$ observe the material is shear thinning. Peng and Wang ${ }^{[26]}$ study the behavior but do not state a conclusion. Here the data in the literature are reanalyzed, aiming to answer the question 'Does shear thickening occur in semisolid metals?'. This paper does not present new experimental results but rather a new, consistent, objective, analysis to rationalize apparent contradictions. The experimental results from the literature have never been compared and contrasted in this systematic way before. The analysis is not dependent on the constitutive equation assumed by the authors of the experimental papers but rather takes their experimental data and deduces whether the behavior is shear thickening or shear thinning, using classical rheological understanding of the definitions of shear thickening

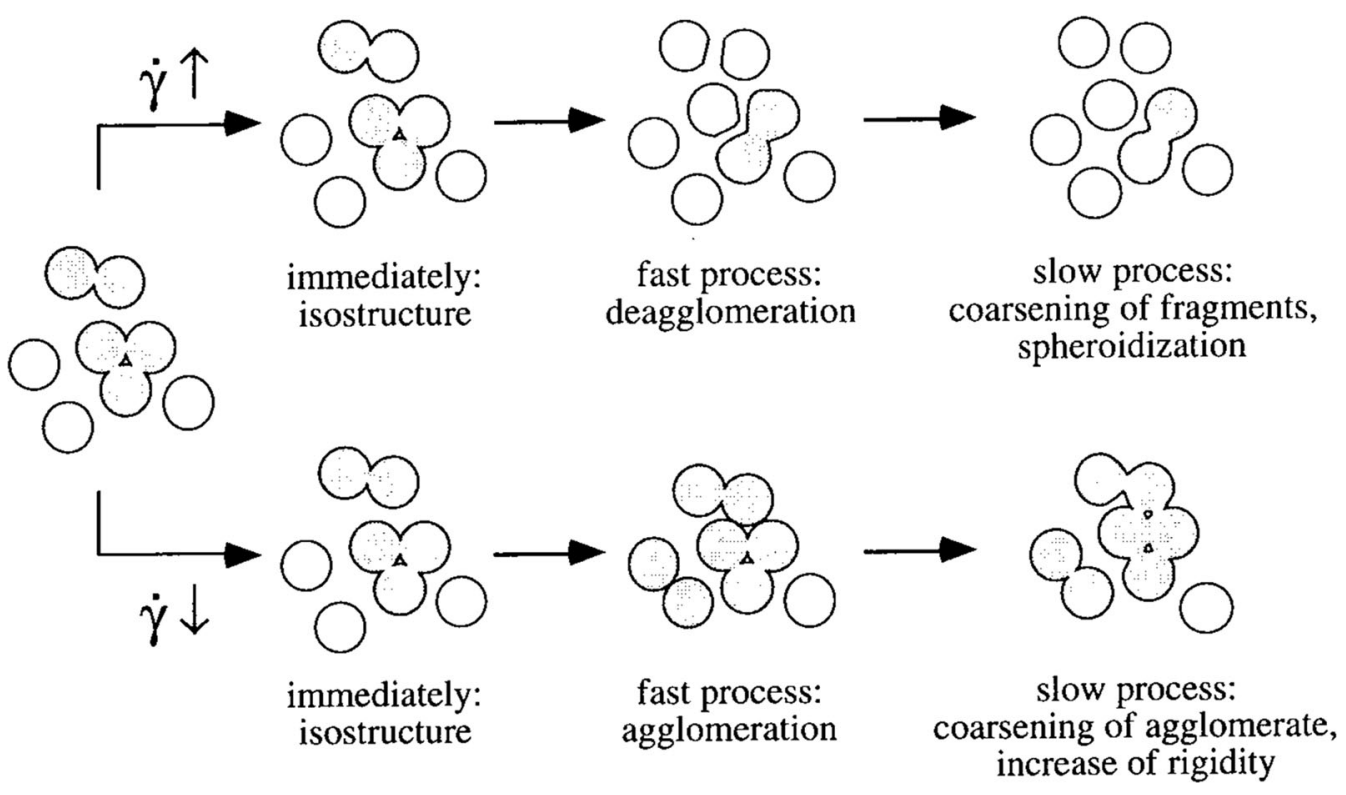

Fig. 2-Schematic diagram ${ }^{[22]}$ showing that when a rapid jump-up in shear rate occurs, immediately after the jump, the materials are 'isostructure' with the starting material. A fast process of deagglomeration (breaking of bonds between spheroids) then occurs. Subsequently, a much slower diffusion controlled process of coarsening and spheroidisation takes place (note the change in shape of particles). When the shear rate jump is down, the processes are a fast process of agglomeration and a slow process of neck growth and coarsening. 
and shear thinning. It is assumed that deformation is homogeneous but the potential existence of a yielded zone is discussed to identify where this is relevant.

\section{ANALYSIS}

In this section, firstly, the experimental results in the literature for rapid shear rate jumps are summarized, and then, the method is given for the new analysis of those results.

\section{A. Experimental Results in the Literature for Rapid Shear Rate Jumps}

The experimental details for experiments on Sn15 pct $\mathrm{Pb}$ (which are concentrated on here as it is the 'model system' for the rheology of semisolid alloys) are summarized in detail in Table I as a series of experimental factors might explain the contradictions. All the results in Table I involve applying jumps in shear rate in cylindrical rheometers over very short time periods and measuring the consequent shear stress response. Table I identifies how each research work has reached its conclusion about whether the behavior is shear thinning or shear thickening as this is critical for what follows. The deliberate strategy here is to keep the extensive detail about the experiments in the literature, and how those authors analyze their data, in the table so that the flow of the argument in the main part of the paper is clear.

\section{B. Method of Analysis in this Paper}

Classical rheological understanding of the definitions of shear thickening and shear thinning means that absolute peak shear stress during the jump should be plotted against the shear rate the jump finishes on (Figure 3). Here, experimental data from the literature papers are used, and the analysis is not dependent on any constitutive equation. The deformation is however assumed to be homogeneous. The right-hand column in Table I identifies how the data for this figure have been obtained from the data in the literature. If the curve has as increasing slope with increasing shear rate, it represents shear-thickening behavior, and a decreasing slope with increasing shear rate is shear thinning. To obtain results which can be compared, a conditioning shear rate (i.e., the shear rate before the jump) has been identified which is common between the results of Koke and Modigell $^{[25]}$ (their Figure 18(b)) and the results of Liu et $a{ }^{[24]}$ (their Table IV). The only common shear rate is $100 \mathrm{~s}^{-1}$. There are no results where the fraction solid is the same. The plot for Koke and Modigell ${ }^{[25]}$ with a fraction solid 0.41 curves upwards and hence is shear thickening. For Liu et al. ${ }^{[24]}$ at a fraction of solid of 0.5 , there is a slight trend upwards, again indicating shear thickening. For a lower fraction of solid of 0.36 from Liu et al., the curve is clearly shear thinning (see Figure 3(b)). Results from Kumar et al. ${ }^{[27]}$ have also been plotted. Although these are not from the common starting shear rate of $100 \mathrm{~s}^{-1}$ (they are based on Figure 2 in Reference 27 paper I), they do allow some examination of trends and again show shear thickening. Liu et al. ' $s^{[24]}$ result for a fraction of solid of 0.36 is therefore the unusual result. This might be explained if there is some transition between 0.36 and 0.41 . The Peng and Wang ${ }^{[26]}$ results then support the hypothesis that there is a transition. Their results are for a conditioning shear rate of $200 \mathrm{~s}^{-1}$. For fractions solid of 0.2 and 0.36 , there is little or no evidence of shear thickening but at fractions solid of 0.46 and 0.5 there is. The other observation from Figure 3(b) is that, for jumps to a shear rate of $100 \mathrm{~s}^{-1}$ or less, where the fraction solid is less than or equal to $\sim 0.36$, shear thickening is not apparent, and for the higher fractions solid, it is hardly discernible. Most of the results for Liu et al. ${ }^{[24]}$ are in this regime which helps to explain their thinking that they do not observe shear thickening.

\section{DISCUSSION}

\section{A. Factors which Might Explain the Discrepancies and Contradictions}

Factors which might explain the discrepancies include the following:

(1) Differences in the shear rate ranges and fractions solid considered;

(2) Whether inertia (both of the measuring head and of the semisolid fluid itself) has been appropriately considered;

(3) The size of the particles in relation to the size of the gap between the inner bob and outer cup of the rheometer;

(4) The existence of a yield stress masking the shear thickening phenomenon as argued by Brown et al. $^{[6]}$

\section{B. Shear Rate Range and Fraction Solid}

The shear rate range for the experiments by Kumar and co-workers ${ }^{[23,27]}$ is higher than that for the experiments by Koke and Modigell ${ }^{[25,28]}$ and Liu et al. ${ }^{[24]}$ (and higher shear rate ranges would be expected to exacerbate shear rate thickening if it is due to hydrodynamics or dilatation). However, the latter two sets of experiments overlap in their shear rate ranges but are still apparently contradictory according to their authors. In terms of fraction solid, Kumar et al. ${ }^{[23,27]}$ give results for 0.45 and above, Peng and Wang ${ }^{[26]}$ for 0.2 through to 0.5 , Koke and Modigell ${ }^{[25]}$ for 0.41 and above, and Liu et al. ${ }^{[24]}$ for 0.36 and 0.5 . For fractions solid of 0.36 and less, the spheroids are quite widely separated and may well be less susceptible to clustering and jamming than for the higher fractions of solid.

\section{Inertia}

There are two types of inertia to consider; that of the measuring head and that of the semisolid fluid itself. Kumar considers inertia in the semisolid fluid in detail in 


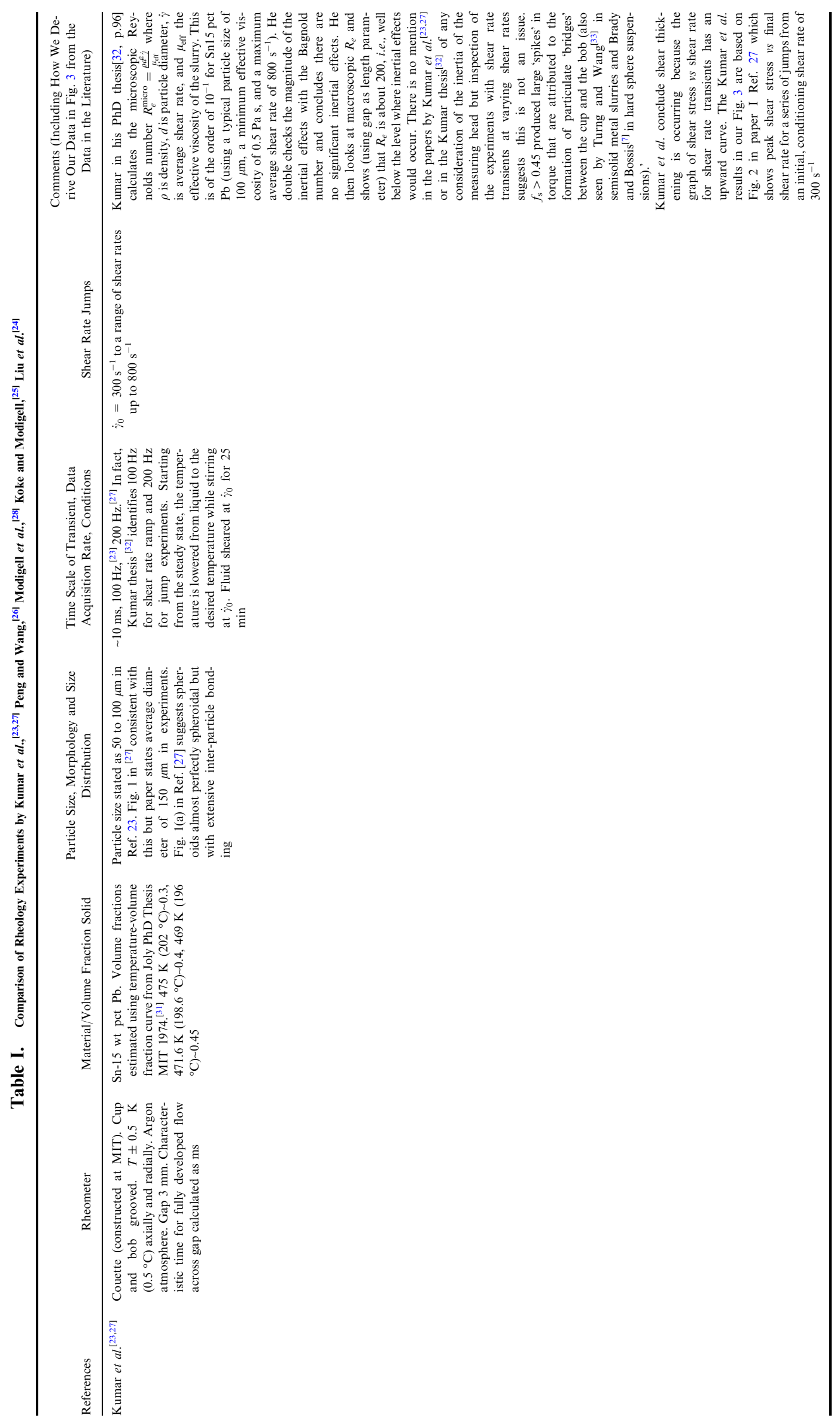




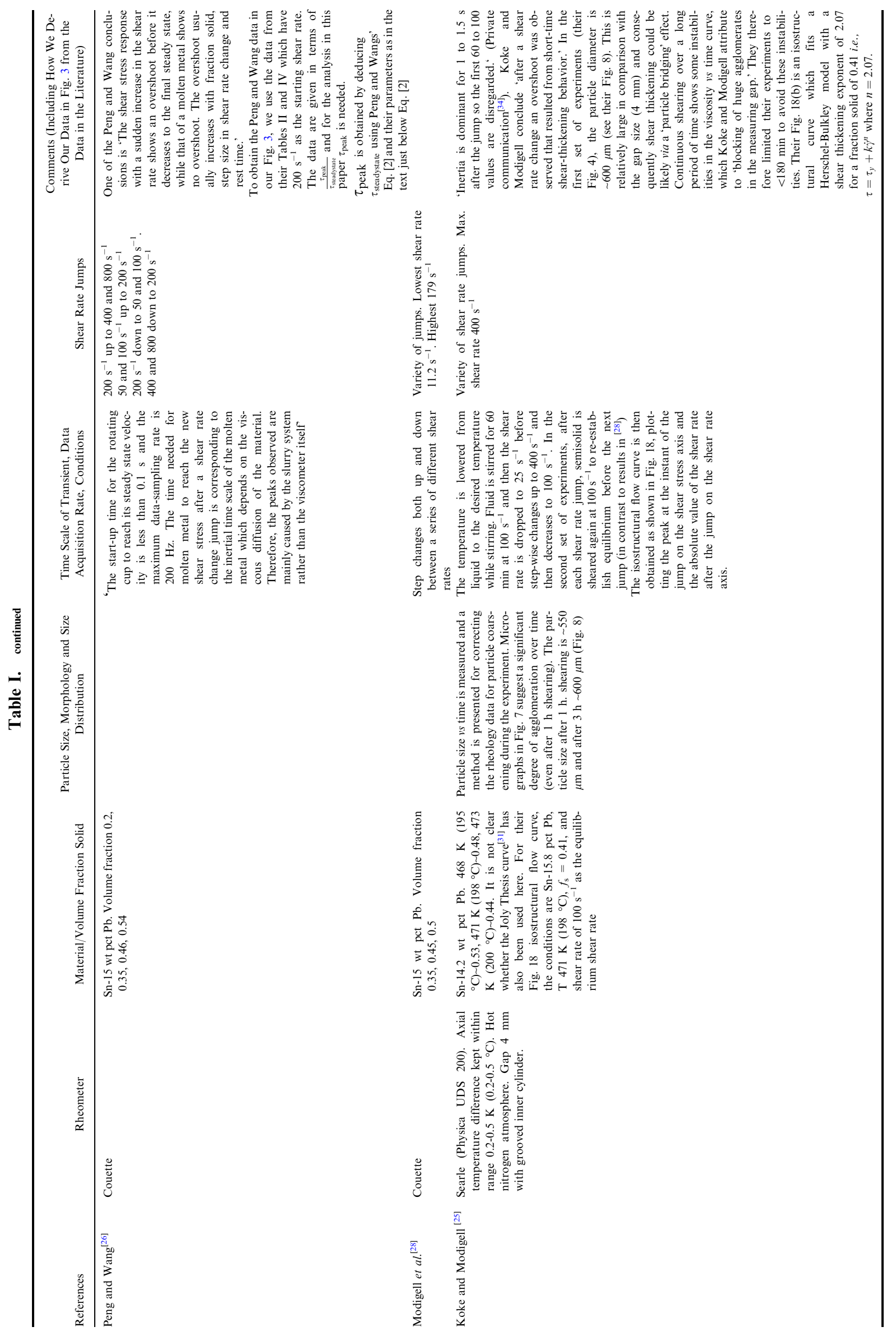




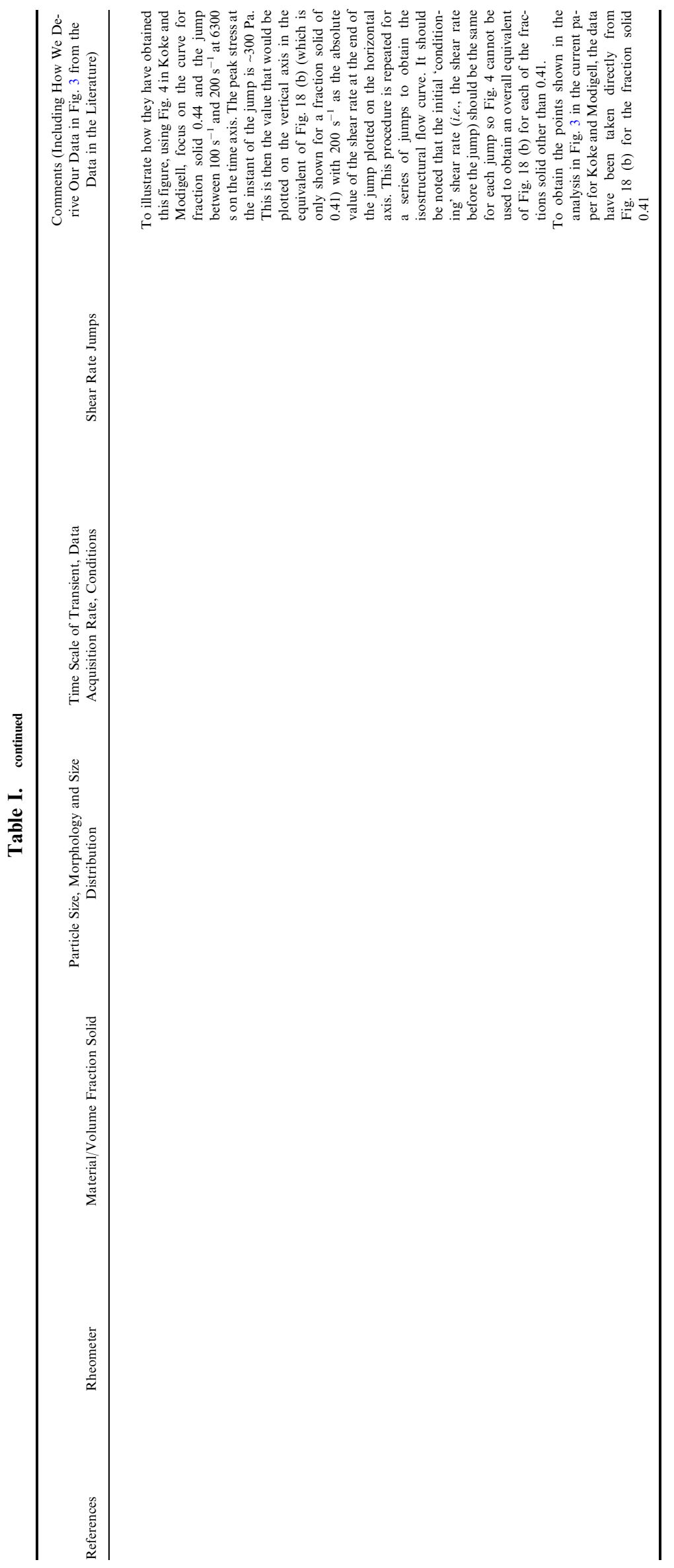

1746 -VOLUME 47A, APRIL 2016

METALLURGICAL AND MATERIALS TRANSACTIONS A 


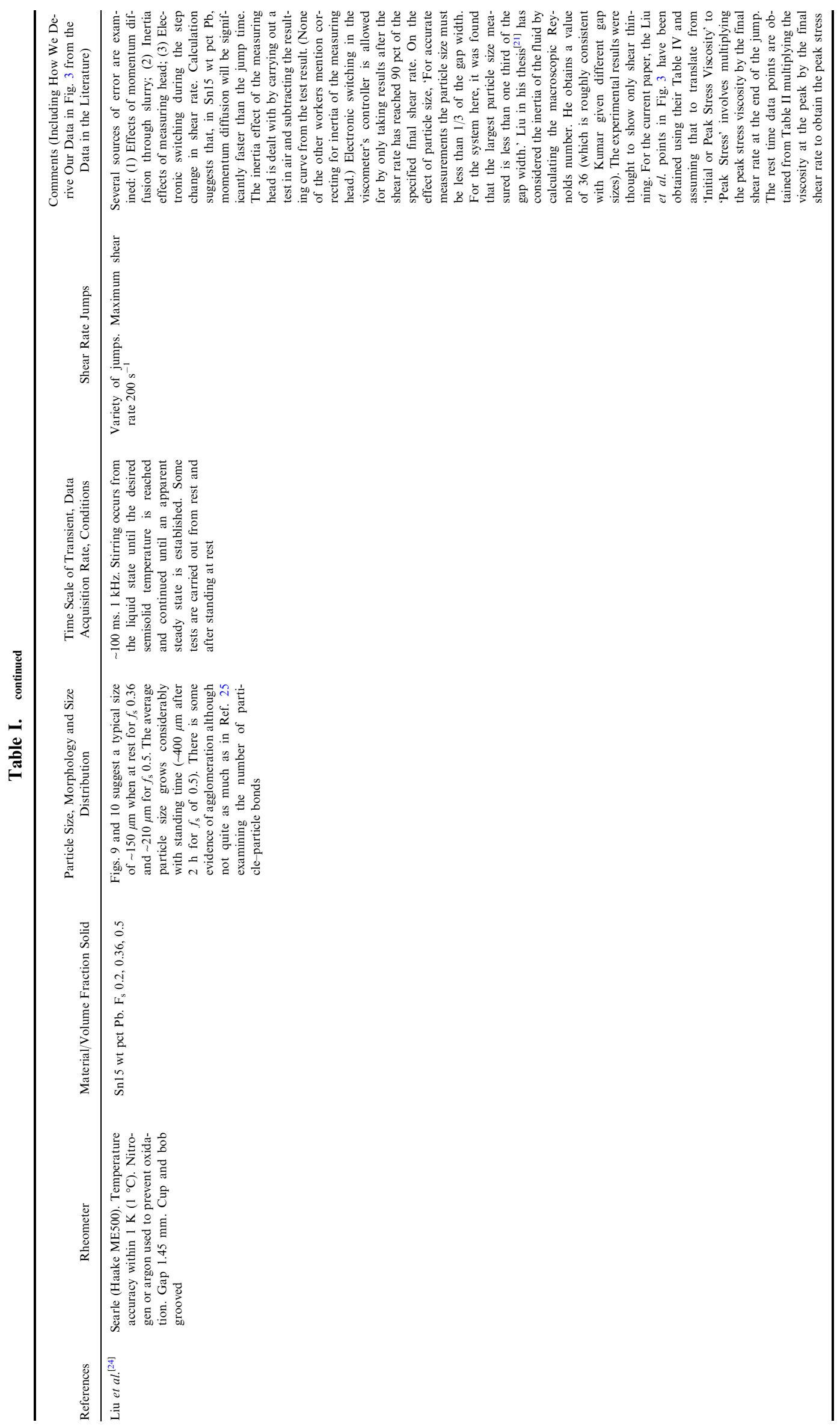




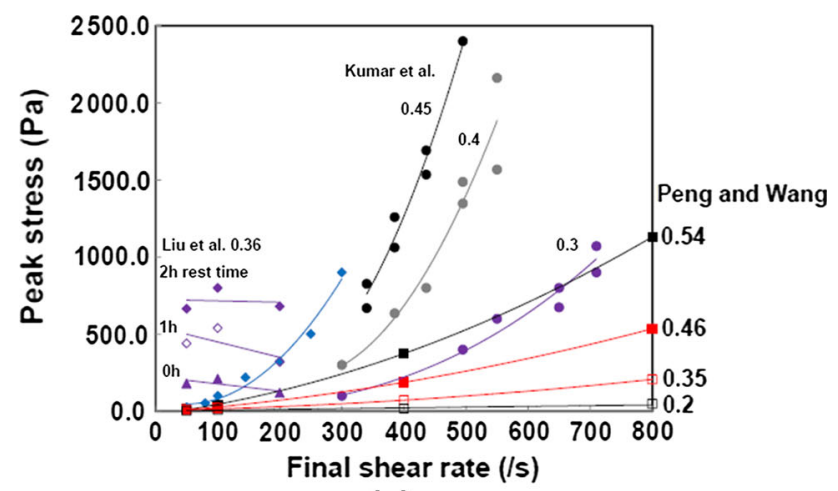

(a)

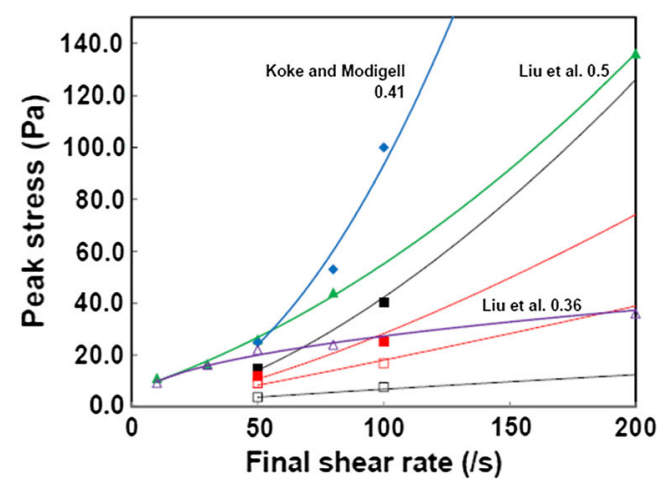

(b)

- $100 / \mathrm{s} ;$ fs $=0.41$ from Koke and Modigell [25] $=200 / \mathrm{s} ;$ fs $=0.54$ from Peng and Wang [26] - $300 \mathrm{ls} ;$ fs $=0.45$ from Kumar et al. [27] $200 \mathrm{ls} ;$ fs $=0.46$ from Peng and Wang [26] $\begin{array}{ll}-300 / \mathrm{s} ; \mathrm{fs}=0.4 \text { from Kumar et al. [27] } & 200 \mathrm{~s} ; \text {; } \mathrm{s}=0.35 \text { from Peng and Wang [26] }\end{array}$

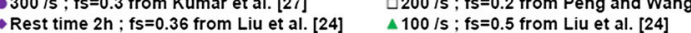
$\Delta$ Rest time $1 \mathrm{~h} ;$ fs $=0.36$ from Liu et al. [24] $\Delta 100 \mathrm{l} ; \mathrm{s} ; \mathrm{fs}=0.36$ from Liu et al. [24] $\Delta$ Rest time oh ; fs $=0.36$ from Liu et al. [24]

Fig. 3-Plot of the peak shear stress during a shear rate jump against the final shear rate after the jump. The shear rate in the legend is the initial, 'conditioning' shear rate. $(b)$ is a magnified version of the bottom left hand corner of $(a)$.

his thesis, ${ }^{[32]}$ looking at microscopic Reynolds number (i.e., on the scale of the particle) and the Bagnold number. He concludes it is safe to ignore the inertial effects in the analysis and confirms this by carrying out shear rate transients at different rates and obtaining results which are essentially unchanged. There is no mention in the papers by Kumar et al. ${ }^{[23,27]}$ or the Kumar thesis ${ }^{[32]}$ of any consideration of the inertia of the measuring head, although the experiments with shear rate transients at varying rates suggest that this is not an issue. Peng and Wang ${ }^{[26]}$ consider that the peaks observed in their work are due to the inertia of the fluid but do not then correct for the effect. Koke and Modigell ${ }^{[25]}$ state 'inertia effects were excluded from the data evaluation.' A subsequent private communication from Modigell ${ }^{[34]}$ identifies that calculation and tests suggest that inertia may be dominant in tin-lead systems for 1 to $1.5 \mathrm{~s}$. Since the sampling rate is $60 \mathrm{~Hz}$, the first 60 to 100 values in the shear stress response were therefore disregarded for the evaluation of the 'isostructure' effect. The rheometer is the same type as in the experiments by Liu et al. ${ }^{[24]}$ (i.e., Searle-type with a rotating bob and static cup), and the plot of shear stress against time in their Figure 7 suggests that the decay in the shear stress after the transient occurs in less than about $0.2 \mathrm{~s}$. In disregarding the first 1 to $1.5 \mathrm{~s}$ of values, the effect of any inertia from the measuring head will also have been dealt with, as the results of Liu et al. ${ }^{[24]}$ suggest that the peak due to this in air (their Figure 5) is dissipated well within $0.2 \mathrm{~s}$. Liu et al. ${ }^{[24]}$ deal with inertia of the measuring head by carrying out an identical test in air to that with the semisolid fluid. A peak in shear stress, immediately after the transient in shear rate is initiated, suggests that inertia is occurring. This is corrected for subsequently, subtracting results for an experiment in air from those for the equivalent shear rate jump with the semisolid fluid. Liu, in his thesis, ${ }^{[21]}$ calculates the Reynolds number for the fluid moving in the gap and concludes that the inertia of the fluid is not an issue. Since inertia of the fluid will increase as the height of the shear rate jump increases, if inertia does exist, and is not corrected for, it will tend to increase the tendency for there to be apparently shear-thickening behavior. However, it can be concluded that inertia has been appropriately dealt with via the various approaches the experimentalists have applied in the literature.

\section{Particle Size and Rheometer Gap Size}

The particle size in Kumar et al.'s experiments is stated to be $\sim 150 \mu \mathrm{m}$ but it is not clear either in the papers $^{[23,27]}$ or in the original thesis by $\operatorname{Kumar}^{[32]}$ that there has been any check for coarsening during the experiments. The gap size is $3 \mathrm{~mm}$ (assumed to mean between the closest surfaces of the cup and the bob i.e., not including the grooves). For Koke and Modigell, [25] the gap size is $4 \mathrm{~mm}$, and the paper itself is focussed on analyzing particle coarsening during shearing and correcting rheological data for the effects of that. At $198 \mathrm{~K}$ (fraction solid, $f_{\mathrm{s}} \sim 0.41$ ), the particle size after 1 hour stirring at $100 \mathrm{~s}^{-1}$ (the 'conditioning' phase prior to the jump) is about $550 \mu \mathrm{m}$. This is significant in relation to the gap size. They observe that, if they continuously shear over a long period of time, some instabilities occur in the viscosity vs time curve. They attribute these to 'blocking of huge agglomerates in the measuring gap' and therefore limit their experiments to shorter time periods where these instabilities are not observed. This is mentioned here because clearly Koke and Modigell have set out to ensure their rapid shear rate jump results are not interfered with by discontinuous shear rate thickening (the instabilities) but they have found it can occur intermittently even under steady-state conditions if the particles are large enough in relation to the gap size. Kumar et al. ${ }^{[23,27]}$ limit their volume fraction solid to 0.45 and less because, above 0.45 , they observed large 'spikes' in torque which they attribute to the formation of particulate 'bridges' between the cup and the bob. The gap size is smallest in the experiments by Liu et al., ${ }^{24]}$ at $1.45 \mathrm{~mm}$. Particle sizes are $\sim 150 \mu \mathrm{m}$ prior to a shear rate jump for $f_{\mathrm{s}} \sim 0.36$ and $\sim 210 \mu \mathrm{m}$ for $f_{\mathrm{s}} \sim 0.5$. Liu et al. argue that for accurate measurements the particle size must be less than $1 / 3$ of the gap width and that their experiments satisfy this requirement. Their results in Figure 3(b) show that there is evidence of shear thickening for a fraction of solid of 0.5 (i.e., the lines curve upwards) but not for the lower fraction of 
solid of 0.36 (where the lines do not show such an upward trend), where the particle size is smaller. Fundamentally, there is an interrelationship between the particle size, the fraction solid, and the gap size with jamming more likely to occur with large particles at high fractions of solid in small gaps.

\section{E. Yield Stress Masking Shear Thickening?}

Figure 3 also shows the results for Liu et al. ${ }^{[24]}$ where the material has been allowed to rest before the jump. Liu et al. ${ }^{[24]}$ identify that there is a clear increase in peak stress with rest time prior to the jump and attribute this to the evolving degree of particle agglomeration. The longer the rest period in the semisolid state prior to semisolid processing, the greater the resistance to deagglomeration in the initial stages of flow. Brown et al. ${ }^{[6]}$ have argued that yield masks shear thickening. This is consistent with the argument in Alexandrou et $a l^{[18,19]}$ about the existence of a yielded zone for low shear rates. However, the evidence suggests that where a conditioning shear rate has been applied (in this case $100 \mathrm{~s}^{-1}$ or above), such yielded zones are not relevant. They only influence the results for shear rate transients if the jump is from rest to a low shear rate with a relatively large gap size.

\section{F. Rationalization}

From these findings, three conclusions are identified in terms of what determines whether shear rate thickening is observed for the transient in shear rate:

(1) Shear rate thickening is not observed if the fraction solid is $\sim 0.36$ or less;

(2) Shear rate thickening is barely discernible if the final shear rate of the jump is in the region of 100 $\mathrm{s}^{-1}$ or less and then only if the fraction solid is above $\sim 0.36$.

(3) Shear rate thickening is not observed if the material has been at rest prior to the jump in shear rate because the yield phenomenon masks any shear thickening tendency;

This analysis can rationalize the apparently contradictory results from Kumar et al., ${ }^{[23,27]}$ Peng and Wang, ${ }^{[26]}$ Koke and Modigell ${ }^{[25]}$, and Liu et al. ${ }^{[24]}$ The reanalysis shows that Liu et al. ${ }^{[24]}$ do have results (for a fraction solid of 0.5 ) which display shear thickening but only when the shear rate jump is occurring from a finite shear rate. If it takes place from rest then the yield stress masks any underlying behavior as discussed by Brown et $a l^{[6]}$ where it is argued that yield can mask shear thickening. For the jumps from a finite shear rate for fractions solid of greater than about 0.36 , an instantaneous shear thickening response is occurring (even if the steady-state behavior is shear thinning) almost certainly for the reasons identified by Brown and Jaeger ${ }^{[12]}$ i.e., the confining stress at boundaries frustrating dilatation.

Figure 3 in Brown and Jaeger ${ }^{[12]}$ is very similar to Figure 3 in this paper when plotted using a log-log scale. This strengthens the argument for our conclusions below. Also in Jiang et al., ${ }^{[35]}$ a suspension of cornstarch was found to exhibit discontinuous shear thickening (such as that we have observed for semisolid metals) for a volume fraction above 0.34 , which is close to the 0.36 value we have been referencing for semisolid metals. As mentioned by Brown and Jaeger, ${ }^{[15]}$ shear thickening starts to gradually appear at a packing fraction of typically around $0.3-0.4$, and the slope on shear stress-shear rate curve increases with increasing volume fraction. ${ }^{[15,35]}$

Jorstad et $a l .{ }^{[36]}$ indicate that in thin sections, solid particles cannot migrate away from the deformation area, so solid-solid interactions increase, resulting in increase in viscosity and so the possibility to have laminar flow at very high velocities. The mechanisms described are very similar to those related to jamming and discontinuous shear thickening. From a practical point of view, shear thickening is therefore potentially relevant for thixoforming at high velocities because it might contribute to ensure laminar flow.

\section{CONCLUSIONS}

There is contradictory evidence in the literature as to whether the behavior during transitions in shear rate for semisolid metals with non-dendritic (i.e., spheroidal) microstructures is shear thinning or shear thickening. Here the data in the literature have been systematically reanalyzed to rationalize the apparent contradictions. It is argued that if the suspension is disagglomerated before the shear rate jump (i.e., it has been initially sheared rather than the jump being from rest), the instantaneous behavior with a jump-up in shear rate is shear thickening (even if the long-term steady-state behavior is shear thinning) provided the fraction solid is greater than about 0.36 and the final shear rate at the end of the jump is greater than about $100 \mathrm{~s}^{-1}$. If the jump-up in shear rate is made from rest then yield masks the shear thickening.

\section{ACKNOWLEDGMENTS}

Professor Atkinson would like to thank Arts et Métiers Paris Tech for their invitation to be a Visiting Professor to carry out this work in collaboration with Professor Favier, and the University of Leicester for permission to visit.

\section{OPEN ACCESS}

This article is distributed under the terms of the Creative Commons Attribution 4.0 International License (http://creativecommons.org/licenses/by/4.0/), which permits unrestricted use, distribution, and reproduction in any medium, provided you give appropriate credit to the original author(s) and the source, provide a link to the Creative Commons license, and indicate if changes were made. 


\section{REFERENCES}

1. H.V. Atkinson: Prog. Mater Sci., 2005, vol. 50, pp. 341-412.

2. D.B. Spencer, R. Mehrabian, and M.C. Flemings: Metall. Trans., 1972, vol. 3, pp. 1925-32.

3. M.C. Flemings: Metall. Trans. A, 1991, vol. 22A, pp. 957-81.

4. V. Favier and H.V. Atkinson: Acta Mater., 2011, vol. 59, pp. 1271-80.

5. HA Barnes: J. Rheol., 1989, vol. 33, pp. 329-66.

6. E. Brown, N.A. Forman, C.S. Orellana, H. Zhang, B.W. Maynor, D.E. Betts, J.M. DeSimone, and H.M. Jaeger: Nat. Mater. Lett., 2010, vol. 9, pp. 220-24.

7. J.F. Brady and G. Bossis: J. Fluid Mech., 1985, vol. 155, pp. 105-29.

8. B.J. Maranzano and N.J. Wagner: J. Chem. Phys., 2001, vol. 114, pp. 10514-527.

9. R.L. Hoffmann: Adv. Colloid Interface Sci., 1982, vol. 17, pp. 161-84.

10. D. Lootens, H. van Damme, Y. Hémar, and P. Hébraud: Phys. Rev. Lett., 2005, vol. 95, Article No. 268302.

11. A. Fall, N. Huang, F. Bertrand, G. Ovarlez, and D. Bonn: Phys. Rev. Lett., 2008, vol. 100, Article No. 018301.

12. E. Brown and H.M. Jaeger: Science, 2011, vol. 333, pp. 1230-31.

13. E. Brown and H.M. Jaeger: J. Rheol., 2012, vol. 56, pp. 875-923.

14. V. Favier, P. Cezard, and R. Bigot: Mater. Sci. Eng., A, 2009, vol. 517, pp. 8-16.

15. E. Brown and H.M. Jaeger: Rep. Prog. Phys., 2014, vol. 77, Article No. 046602.

16. P.C.F. Møller, J. Mewis, and D. Bonn: Soft Matter, 2006, vol. 2, pp. 274-83.

17. A.R.A. McLelland, N.G. Henderson, H.V. Atkinson, and D.H. Kirkwood: Mater. Sci. Eng., A, 1997, vol. A232, pp. 110-18.

18. A.N. Alexandrou and G. Georgiou: J. Non-Newton. Fluid Mech., 2007, vol. 142, pp. 199-206.

19. A.N. Alexandrou, N. Constantinou, and G. Georgiou: $J$. Non-Newton. Fluid Mech., 2009, vol. 158, pp. 6-17.
20. CM Gourlay and AK Dahle: Nature, 2007, vol. 445, pp. 70-73.

21. T.Y. Liu: PhD Thesis, University of Sheffield, 2002.

22. C.J. Quaak: PhD Thesis, Technische Universiteit Delft, 1996, ISBN 90-5651-019-3.

23. P. Kumar, C.L. Martin, and S. Brown: Metall. Trans. A, 1993, vol. 24A, pp. 1107-16.

24. T.Y. Liu, H.V. Atkinson, P.J. Ward, and D.H. Kirkwood: Metall. Mater. Trans. A, 2003, vol. 34A, pp. 409-17.

25. J. Koke and M. Modigell: J. Non-Newton. Fluid Mech., 2003, vol. 112 , pp. 141-60.

26. H. Peng and K.K. Wang: in Proc. 4th Int. Conf. on Semi-Solid Processing of Alloys and Composites, D.H. Kirkwood and P. Kapranos, eds., Publ. University of Sheffield, Sheffield, UK, June 1996, pp. 2-9.

27. P. Kumar, C.L. Martin, and S. Brown: Acta Metall. Mater., 1994, vol. 42, pp. 3595-602 and 3603-14.

28. M. Modigell, J. Koke, and J. Petera: in Proc. 5th Int. Conf. on Semi-solid Processing of Alloys and Composites, A.K. Bhasin, J.J. Moore, K.P. Young, and S. Midson, eds., Publ. Colorado School of Mines, Golden, Colorado, USA, June 1998, pp. 317-326.

29. G.R. Burgos, A.N. Alexandrou, and V. Entov: J. Mater. Process. Technol., 2001, vol. 110, pp. 164-76.

30. H.V. Atkinson and P.J. Ward: JOM, 2006, vol. 8, pp. 21-23.

31. P.A. Joly: PhD Thesis, Massachusetts Institute of Technology, Cambridge, MA, 1974.

32. P. Kumar: PhD Thesis, Massachusetts Institute of Technology, Cambridge, MA, 1994.

33. L.S. Turng and K.K. Wang: J. Mater. Sci., 1991, vol. 26, pp. $2173-83$.

34. M. Modigell: AVT at RWTH Aachen University, Germany, and German University of Technology in Oman (GUTech), Private Communication, July 2011.

35. W. Jiang, S. Xuan, and X. Gong: Appl. Phys. Lett., 2015, vol. 106, Article number 151902 .

36. J.L. Jorstad, A.N. Alexandrou, and E. Mitsoulis: Solid State Phenom., 2015, vols. 217-218, pp. 159-65. 